\title{
Two-hit model of brain damage in the very preterm newborn: small for gestational age and postnatal systemic inflammation
}

\author{
Alan Leviton', Raina N. Fichorova ${ }^{2}$, T. Michael O'Shea ${ }^{3}$, Karl Kuban ${ }^{4-6}$, Nigel Paneth ${ }^{7,8}$, Olaf Dammann ${ }^{9}$ and Elizabeth N. Allred ${ }^{1,10}$; \\ for the ELGAN Study Investigators
}

BACKGROUND: We sought to disentangle the contributions of perinatal systemic inflammation and being small for gestational age (SGA) to the occurrence of low Bayley Mental Development Indices (MDIs) at the age of $2 \mathrm{y}$.

METHODS: We measured the concentration of 25 inflammation-related proteins in blood obtained during the first two postnatal weeks from 805 infants who were born before the 28th wk of gestation and who had MDI measurements at the age of $2 y$ and were able to walk independently.

RESULTS: SGA newborns who did not have systemic inflammation (a concentration of an inflammation-related protein in the top quartile for gestational age on two days a week apart) were at a greater risk of an $\mathrm{MDI}<55$, but not 55-69, than their peers who had neither SGA nor systemic inflammation. SGA infants who had elevated blood concentrations of interleukin (IL)-1 $\beta$, tumor necrosis factor-a, or IL-8 during the first 2 postnatal weeks were at even higher risk of an $\mathrm{MDI}<55$ than their SGA peers without systemic inflammation and their non-SGA peers with systemic inflammation.

CONCLUSION: SGA appears to place very preterm newborns at an increased risk of a very low MDI. Systemic inflammation adds considerably to the increased risk.

B eing small for gestational age (SGA) occurs more commonly among very preterm newborns than among their peers born closer to term (1). SGA preterm infants are more likely than their more appropriately grown peers to have structural abnormalities of the brain (2). They are also more likely to have multiple expressions of developmental delay/limitations, including low scores on cognition assessments $(3,4)$. These abnormalities are compatible with early direct insults to the brain or disturbances to developmental processes.

Systemic inflammation also places preterm infants at an increased risk of functional limitations and indicators of reduced brain volume (5-13).

In a two-hit model of brain damage, SGA appeared to sensitize neonatal rats to a subsequent inflammatory stimulus, resulting in more brain damage than that seen in control rats following the same inflammatory stimulus (14). We are not aware of any report of an assessment of such a two-hit model in extremely low gestational age human newborns. Such an assessment might help elucidate how much of the brain damage in SGA preterm newborns can be attributed to growth restriction and how much to subsequent inflammation.

\section{RESULTS}

Sample Description

Of the 805 infants born before the 28th wk of gestation who had inflammation-related proteins measured on $2 \mathrm{~d}$ in perinatal blood spots, a Bayley Scales of Infant Development assessment at the age of $2 \mathrm{y}$ and a Gross Motor Function Classification System score $<1$ at $2 y, 11 \%(n=91)$ had a Mental Development Index (MDI) $<55$, which is $\sim 85$-fold the expected $0.13 \%$ for a score $>3$ SDs below the expected mean (Table 1). Another 85 children had an MDI in the 55-69 range, which places the MDI at $>2$ SDs below the expected mean but above the 3 SDs criterion. The sample consisted of these 176 children plus their 629 peers with higher MDI scores who also were able to walk and had proteins measured in perinatal blood spots.

\section{Potential Confounders: Delivery and Placenta Characteristics}

We sought characteristics and exposures that might distort an assessment of the relationships between each of the antecedents of interest (i.e., SGA and postnatal systemic inflammation) and the outcomes of interest, an MDI $<55$ and an MDI between 55 and 69, in light of the contribution of the other antecedents. Women who had severe preeclampsia were more likely than others to give birth to an SGA infant (55 vs. $8 \%$ for prelabor rupture of membranes and 7\% for preterm labor) and to infants with a very low MDI ( 17 vs. $14 \%$ for prelabor rupture of membranes and 9\% for preterm labor) (Table 2). Infants born to these women were not at increased risk of having persistent or recurring elevated blood concentrations of inflammation-related proteins. Delivery for a fetal indication was also associated

'Department of Neurology, Boston Children's Hospital and Harvard Medical School, Boston, Massachusetts; ${ }^{2}$ Department of Obstetrics, Gynecology and Reproductive Biology, Brigham and Women's Hospital and Harvard Medical School, Boston, Massachusetts; ${ }^{3}$ Department of Pediatrics, Wake Forest School of Medicine, Winston-Salem, North Carolina; ${ }^{4}$ Department of Pediatrics, Boston Medical Center, Boston, Massachusetts; ${ }^{5}$ Department of Pediatrics, Boston University School of Medicine, Boston, Massachusetts; ${ }^{6}$ Department of Neurology, Boston University School of Medicine, Boston, Massachusetts; ${ }^{7}$ Department of Epidemiology and Biostatistics, Michigan State University, East Lansing, Michigan; ${ }^{8}$ Department of Pediatrics and Human Development, Michigan State University, East Lansing, Michigan; ${ }^{9}$ Department of Public Health and Community Medicine, Tufts University School of Medicine, Boston, Massachusetts; ${ }^{10}$ Department of Biostatistics, Harvard School of Public Health, Boston, Massachusetts. Correspondence: Alan Leviton (alan.leviton@childrens.harvard.edu) 
Table 1. Sample description

\begin{tabular}{lc}
\hline & $\begin{array}{c}\text { Number of } \\
\text { newborns }\end{array}$ \\
\hline Enrolled & 1,506 \\
Survived to $2 y$ & 1,200 \\
Bayley Scales of Infant Development assessment at the & 1,018 \\
age of 2 y & \\
Gross Motor Function Classification System score $<1$ at 2 y & 921 \\
Proteins measured in blood collected on 2 or 3 d & 805 \\
Mental Development Index (MDI) $\geq 70$ & 629 \\
MDI $\geq 55,<70$ & 85 \\
$M D I<55$ & 91
\end{tabular}

with both an MDI in the 55-69 range and persistent/recurrent elevated concentrations of interleukin (IL)- $1 \beta$, IL-8, and intracellular adhesion molecule (ICAM)-1. Receipt of magnesium for seizure prophylaxis (a correlate of preeclampsia) was associated with both SGA and an MDI < 55 but not with elevated concentrations of inflammation-associated proteins.

Infants whose placenta harbored an organism or had histologic inflammation of the membranes or umbilical cord were less likely than others to have been SGA but were not at increased risk of an MDI $<55$. Those whose placenta and umbilical cord had histologic inflammation were more likely than others to have elevated concentrations of inflammationrelated proteins.

Table 2. The distribution of intrauterine growth restriction and postnatal systemic inflammation in categories of delivery and placenta characteristics

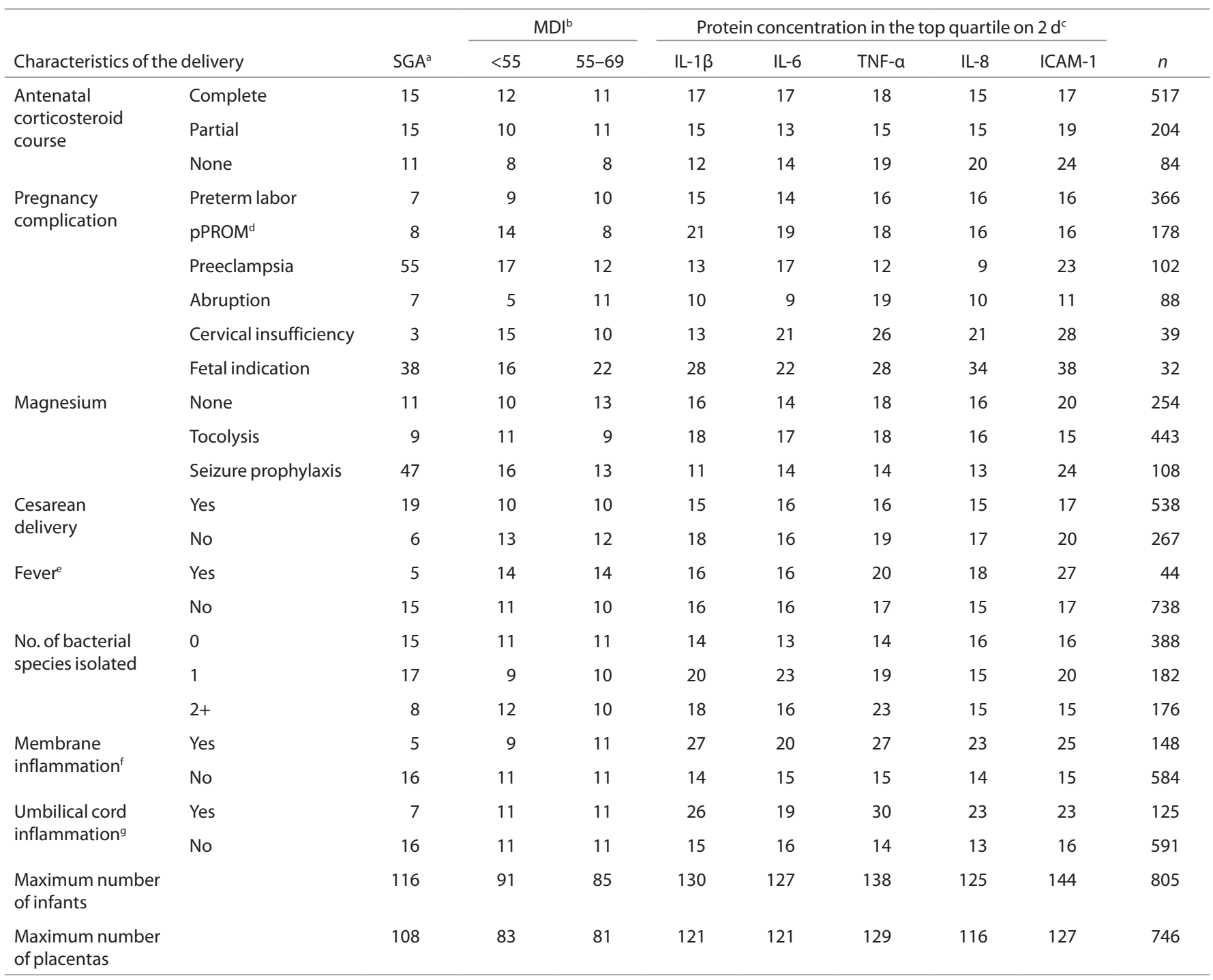

These are row percentages.

ICAM, intercellular adhesion molecule; IL, interleukin; MDI, Mental Development Index; pPROM, preterm, premature rupture of fetal membranes; SGA, small for gestational age; TNF, tumor necrosis factor.

airth weight below the 10th percentile for gestational age. ${ }^{b}$ Bayley Scales of Infant Development Mental Development Index when Gross Motor Function Classification System

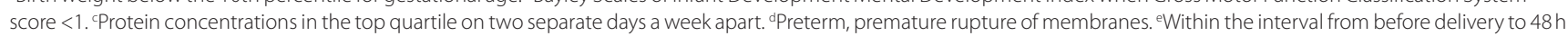
postdelivery. ${ }^{\mathrm{f}}$ Membrane inflammation is defined as inflammation of the chorionic plate (stage 3 and severity 3 ) or of the chorion/decidua (moderate or severe). ${ }^{9}$ inflammation of the umbilical cord grade 3 or higher, which requires neutrophils in perivascular Wharton's jelly. 
Table 3. The distribution of intrauterine growth restriction and postnatal systemic inflammation in categories of the newborn's characteristics

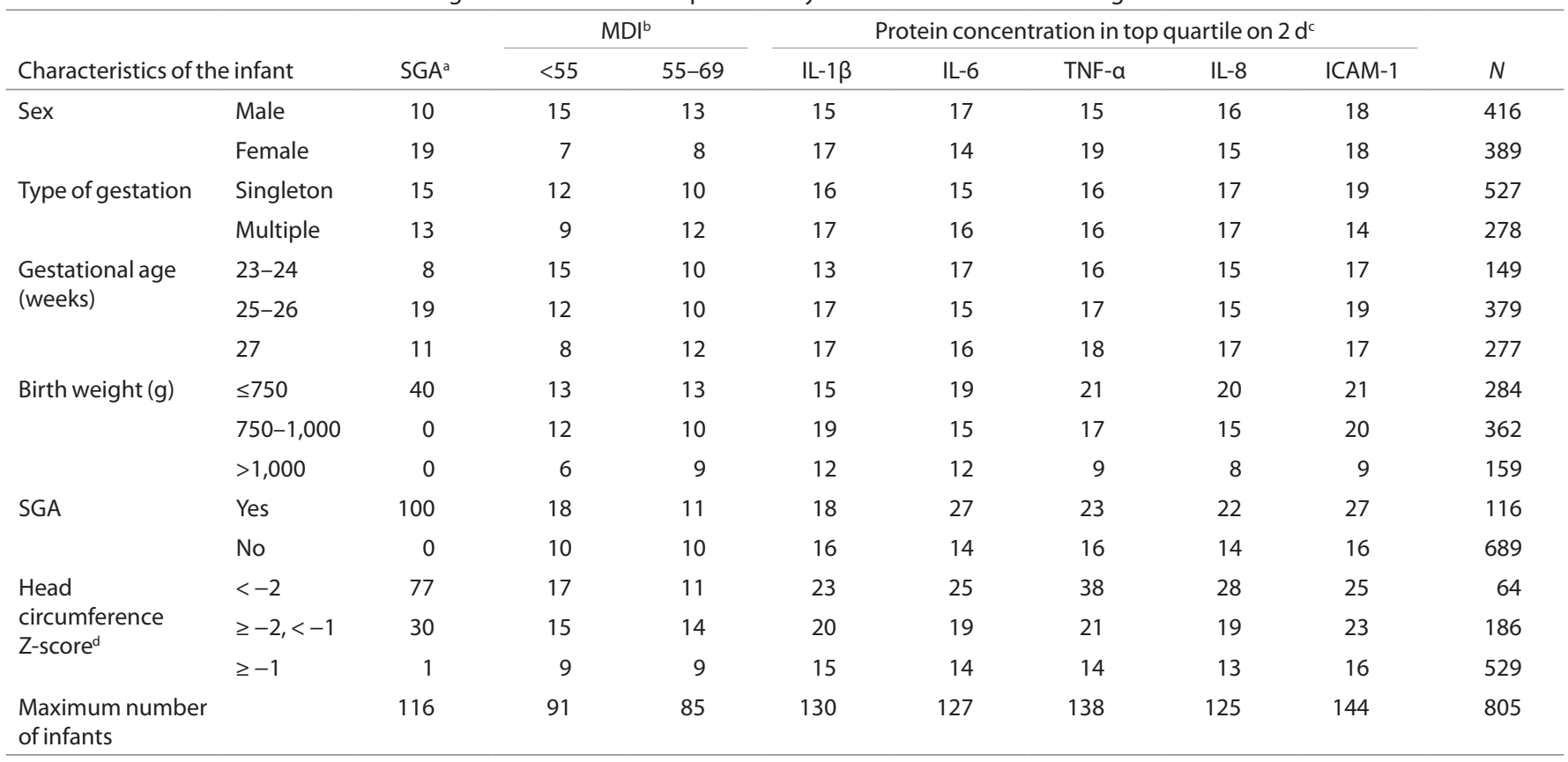

These are row percentages.

ICAM, intercellular adhesion molecule; IL, interleukin; MDI, Mental Development Index; SGA, small for gestational age; TNF, tumor necrosis factor.

aBirth weight below the 10th percentile for gestational age. ${ }^{b}$ Bayley Scales of Infant Development Mental Development Index when Gross Motor Function Classification System score $<1$. 'Protein concentrations in the top quartile on two separate days a week apart. ${ }^{~}$ Yudkin standard.

\section{Potential Confounders: Characteristics of the Newborn}

Boys were less likely than girls to be SGA (10 vs. 19\%) and more likely to have an MDI <55 and an MDI between 55 and 69 (Table 3). The lower the gestational age, the higher the rate of very low MDI. SGA infants were more likely than others to have had elevated concentrations of IL- 6 , tumor necrosis factor- $\alpha$ (TNF- $\alpha$ ), IL-8, and ICAM-1 on two separate days during the first 2 wk after birth. The smaller the head circumference at birth, the higher the rate of very low MDI and of elevated blood concentrations of inflammation-related proteins on two separate days.

\section{Potential Confounders: Postnatal Characteristics}

Infants who had hyperoxemia, hypercarbia, or acidemia on two of the first three postnatal days were more likely than others to be SGA but not appreciably more likely to have a very low MDI, although newborns with hypercarbia or acidemia were at increased risk of an MDI in the 55-69 range (Table 4). Newborns who had early postnatal acidemia were more likely than others to be SGA and have prominent systemic inflammation. Bacteremia was associated with both an MDI $<55$ and systemic inflammation but not with SGA. Infants who were ventilated on days 7, 14, and 21 were more likely than others to be SGA and have low MDIs and systemic inflammation. An MDI in the 55-69 range was minimally associated with both late bacteremia and ventilation.

Influence of the Co-occurrence of SGA and Inflammatory Stimuli on Low MDI

The risk of an MDI < 55 was highest in SGA infants who had bacteremia during the second, third, or fourth weeks, and the risk of an MDI between 55 and 69 was elevated among SGA infants who had bacteremia during the first postnatal week (Table 5). The risk of an MDI <55 was also elevated in SGA infants who were ventilated on postnatal day 7 or later. By contrast, however, ventilation did not appear to have a disproportionate effect on SGA infants.

Odds Ratios of a Very Low or Moderately Low MDI Among Children Classified by Whether or Not They Were SGA and by Protein Concentration Elevations

Because we constructed a separate model for each protein, Table 6 displays the results obtained with 25 separate models.

\section{$\mathrm{MDI}<55$}

As compared with children who had neither SGA nor an elevated concentration of the individual protein on two days separated by $\sim 1$ wk (fourth data column), those who were SGA, but without repeatedly high concentrations of an individual protein, had a fold increase in risk of an MDI $<55$ in the range of 1.7-3.8, with most odds ratios significant at $P<0.01$ (third data column) (Table 6). In essence, these infants were at an increased risk, regardless of the protein assessed. Their increased risk is associated with being SGA and not with systemic inflammation. The odds ratios vary for different proteins because the risk of a very low MDI associated with being SGA in these models is influenced by the effect of elevated protein concentrations on the risk among other infants. By contrast, those SGA babies who had repeatedly high concentrations of serum amyloid A (SAA), IL-1 $\beta$, IL-6, TNF- $\alpha$, IL-8, macrophage inflammatory protein-1 $\beta$, ICAM-1, E-selectin, and/or insulin growth factor binding protein-1 were at a much higher risk of a very low MDI (first data column). Most of the odds ratios for these children were double 
Table 4. The distribution of intrauterine growth restriction and postnatal systemic inflammation in categories of the newborn's early postnatal characteristics

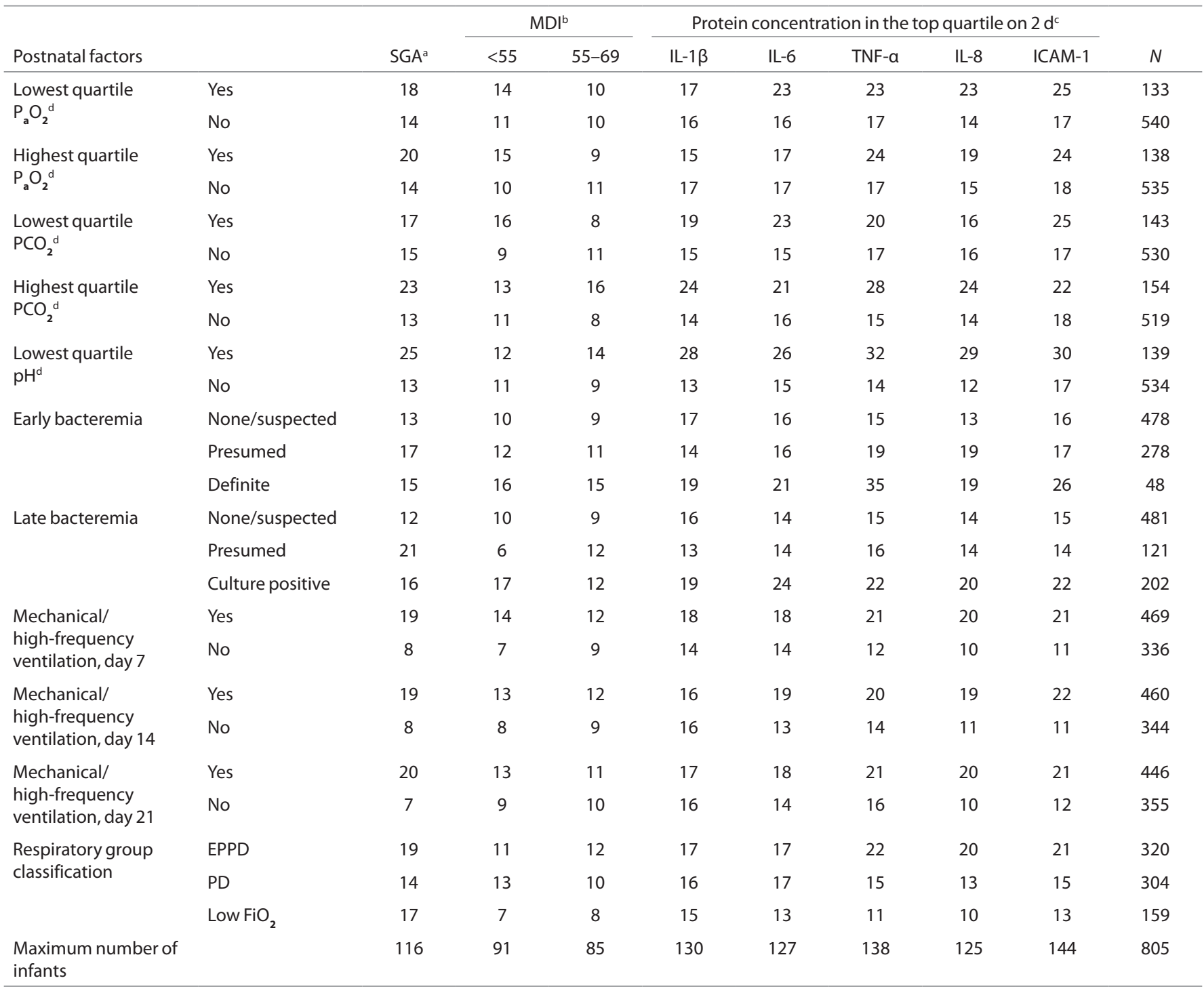

These are row percentages.

EPPD, early and persistent pulmonary dysfunction; $\mathrm{FiO}_{2}$, fraction of inspired oxygen; ICAM, intercellular adhesion molecule; IL, interleukin; MDI, Mental Development Index; PD, pulmonary deterioration; SGA, small for gestational age; TNF, tumor necrosis factor.

aBirth weight below the 10th percentile for gestational age. ${ }^{b}$ Bayley Scales of Infant Development Mental Development Index when Gross Motor Function Classification System score $<1$. CProtein concentrations in the top quartile on two separate days a week apart. ${ }^{~}$ Extreme quartile for gestational age on 2 of the first 3 postnatal $d$.

those of their SGA peers who did not have repeatedly elevated concentrations of these proteins (third data column). The risks of a very low MDI were significantly increased in infants who were not SGA but did have recurrent/persistent elevated concentrations of C-reactive protein (CRP), SAA, IL-8, ICAM-1, E-selectin, and vascular endothelial growth factor receptor-2 (second data column). These odd ratios were not as high as those seen among SGA infants who had systemic inflammation.

In separate analyses (data not shown), only bacteremia, and not the indication for delivery or duration of ventilation, contributed to the risk of an MDI <55, without appreciably reducing the risks associated with being SGA and indicators of systemic inflammation. The odds ratios for bacteremia were close to 2.0 and were statistically significant at $P<0.01$.
No interaction was seen between being SGA and bacteremia, delivery indication, or duration of ventilation.

$\mathrm{MDI}=55-69$

SGA infants who did not have repeatedly high concentrations of an individual protein were not at appreciably increased risk of an MDI in the 55-69 range (Table 6, penultimate column). Among SGA infants who had repeatedly high concentrations of individual proteins, SAA was the only protein to achieve statistical significance (third data column from the right). Although repeatedly high concentrations of several proteins were associated with increased risk of an MDI in the 55-69 range among SGA infants, only macrophage inflammatory protein- $1 \beta$ had a statistically significant elevated odds ratio (fourth data column from the right). 


\section{Articles | Leviton et al.}

Table 5. Percentage of children who had both the row and column characteristics who had an MDI $<55$ or an MDI between 55 and 69

\begin{tabular}{|c|c|c|c|c|c|}
\hline \multirow[b]{3}{*}{ Postnatal factors } & & \multicolumn{2}{|c|}{$\mathrm{MDI}<55^{\mathrm{a}}$} & \multirow{2}{*}{\multicolumn{2}{|c|}{$\begin{array}{c}\text { MDI between } 55 \text { and } 69^{\mathrm{a}} \\
\mathrm{SGA}^{\mathrm{b}}\end{array}$}} \\
\hline & & \multicolumn{2}{|c|}{$S G A^{b}$} & & \\
\hline & & Yes & No & Yes & No \\
\hline \multirow{2}{*}{ Bacteremia during the first week } & Presumed & 17 & 12 & 15 & 10 \\
\hline & Culture positive & 14 & 17 & 29 & 12 \\
\hline \multirow[t]{2}{*}{ Bacteremia during weeks 2-4 } & None/suspected & 12 & 9 & 15 & 9 \\
\hline & Culture positive & 34 & 14 & 3 & 14 \\
\hline \multirow[t]{2}{*}{ Mechanical/high-frequency ventilation, day 7} & Yes & 22 & 12 & 12 & 12 \\
\hline & No & 4 & 8 & 7 & 9 \\
\hline \multirow[t]{2}{*}{ Mechanical/high-frequency ventilation, day 14} & Yes & 20 & 12 & 13 & 12 \\
\hline & No & 14 & 8 & 7 & 9 \\
\hline
\end{tabular}

These are cell-specific percentages.

MDI, Mental Development Index; SGA, small for gestational age.

aBayley Scales of Infant Development Mental Development Index when Gross Motor Function Classification System score $<1$. bBirth weight below the 10th percentile for gestational age.

\section{DISCUSSION}

Our main finding was that extremely preterm SGA newborns were at increased risk of a very low MDI $(<55)$. When they had systemic inflammation in addition to being SGA, their risks were considerably higher than the risks of infants who had systemic inflammation and were not SGA, infants who were SGA and did not have inflammation, as well as infants who had neither inflammation nor SGA. In essence, SGA alone or phenomena associated with SGA appear to place SGA newborns at an increased risk, and this risk is prominently heightened if they were also exposed to prolonged or repetitive systemic inflammation during the first $2 \mathrm{wk}$ after birth.

\section{Limitations and Strengths}

The weaknesses of our study are those of all observational studies. We are unable to distinguish between causation and association as explanations for what we found.

Our study has several strengths. First, we selected infants on the basis of gestational age, not birth weight, in order to minimize confounding due to factors related to fetal growth restriction (15). Second, we collected all of our data prospectively. Third, examiners were not aware of the medical histories of the children they examined, thereby minimizing "diagnostic suspicion bias" (16). Fourth, we have minimized observer variability as best we can in the assessments of neurodevelopmental functions (17). Fifth, attrition in the first $2 \mathrm{y}$ was modest. Sixth, our protein data are of high quality (18) and have high content validity (19-22).

\section{Maternal and Fetal Indications for Delivery, SGA, and Inflammation}

Although preeclampsia might not be an inflammatory disorder primarily, many characteristics and associations link it to inflammatory phenomena (23). Thus, the first hit might have been exposure to maternal inflammation (24).
SGA infants tended to have higher blood concentrations of IL-6, TNF- $\alpha$, IL-8, and ICAM-1 on repeated occasions than others. Nevertheless, although $55 \%$ of infants born to preeclamptic women were SGA, they were not more likely than others to have repeated elevations of blood concentrations of inflammation-associated proteins. By contrast, infants delivered for fetal indications tended to have prominently elevated concentrations of IL- $1 \beta$, IL-8, and ICAM-1, even though only $38 \%$ of these newborns were SGA. Perhaps the magnesium given to preeclamptic women dampened the inflammatory responses of their SGA newborns (25).

\section{SGA, Postnatal Systemic Inflammation, and Very Low MDI}

The odds ratios of an MDI <55 associated with persistent/ recurrent elevated concentrations of SAA, IL-1 $\beta$, IL-6, TNF- $\alpha$, IL- 8 , ICAM-1, E-selectin, and insulin growth factor binding protein-1 were considerably higher among SGA children than among their peers. These observations are compatible with two possibilities. One is that processes associated with being SGA and processes associated with systemic inflammation each contribute independently to the risk of a very low MDI. The other is that processes associated with SGA sensitize the brain to the adverse effects of postnatal inflammation.

\section{Two-Hit Models of Brain Damage}

"Morbidities associated with preterm delivery appear to be additive to those associated with fetal growth restriction so SGA, preterm infants may be at great risk for poor neurodevelopmental outcome" (26). This quote exemplifies the concept of the greater the number of risk factors, the higher the risk. On the other hand, the heightened risk might not be additive.

This two-hit model is exemplified by the greater extent of brain damage following intracerebral lipopolysaccharide 
Table 6. Odds ratios (and $99 \%$ confidence intervals) of an $\mathrm{MDI}<55$ or an MDI between 55 and 69 vs. those with an $\mathrm{MDI} \geq 70$

\begin{tabular}{|c|c|c|c|c|c|c|c|c|}
\hline \multirow[b]{5}{*}{ Protein } & \multicolumn{4}{|c|}{$\mathrm{MDI}^{\mathrm{a}}<55$} & \multicolumn{4}{|c|}{$\mathrm{MDI}^{\mathrm{a}}$ between 55 and 69} \\
\hline & \multicolumn{8}{|c|}{ Protein concentration in top quartile on two separate days a week apart } \\
\hline & \multicolumn{2}{|c|}{ Yes } & \multicolumn{2}{|l|}{ No } & \multicolumn{2}{|c|}{ Yes } & \multicolumn{2}{|l|}{ No } \\
\hline & \multicolumn{4}{|c|}{$S G A^{b}$} & \multicolumn{4}{|c|}{$S_{G} A^{b}$} \\
\hline & Yes & No & Yes & No & Yes & No & Yes & No \\
\hline CRP & $2.2(0.5,9.5)$ & $3.2(1.5,6.7)$ & $3.8(1.6,8.9)$ & 1.0 & $1.6(0.4,7.1)$ & $1.6(0.7,3.7)$ & $1.5(0.5,4.2)$ & 1.0 \\
\hline SAA & $6.6(1.6,27)$ & $3.3(1.7,7.1)$ & $2.7(1.1,6.5)$ & 1.0 & $1.7(0.2,13)$ & $2.2(1.01,5.0)$ & $1.6(0.6,4.2)$ & 1.0 \\
\hline MPO & $2.6(0.5,14)$ & $1.1(0.5,2.6)$ & $2.6(1.2,5.9)$ & 1.0 & $0.7(0,9.8)$ & $0.7(0.3,1.8)$ & $1.5(0.6,3.7)$ & 1.0 \\
\hline IL-1 $\beta$ & $4.7(1.2,19)$ & $1.7(0.7,3.8)$ & $2.4(1.02,5.7)$ & 1.0 & $1.4(0.2,10)$ & $1.0(0.4,2.6)$ & $1.4(0.6,3.7)$ & 1.0 \\
\hline IL-6 & $6.9(2.3,21)$ & $1.9(0.9,4.4)$ & $1.7(0.6,4.6)$ & 1.0 & $2.2(0.5,9.6)$ & $1.1(0.4,2.7)$ & $1.3(0.5,3.4)$ & 1.0 \\
\hline IL-6R & $2.3(0.4,13)$ & $1.3(0.6,2.8)$ & $2.7(1.2,6.2)$ & 1.0 & $1.8(0.3,10)$ & $0.8(0.3,1.9)$ & $1.3(0.5,3.3)$ & 1.0 \\
\hline TNF-a & $6.9(2.0,24)$ & $2.0(0.9,4.6)$ & $2.1(0.9,4.3)$ & 1.0 & $3.5(0.8,14)$ & $1.5(0.7,3.5)$ & $1.1(0.4,3.2)$ & 1.0 \\
\hline TNF-R1 & $1.2(0.2,8.9)$ & $1.1(0.5,2.6)$ & $3.0(1.3,6.7)$ & 1.0 & $2.6(0.6,10)$ & $0.6(0.2,1.7)$ & $1.0(0.4,2.9)$ & 1.0 \\
\hline TNF-R2 & $2.5(0.4,14)$ & $1.8(0.8,4.0)$ & $2.9(1.3,6.6)$ & 1.0 & $3.3(0.7,15)$ & $1.9(0.9,4.1)$ & $1.3(0.5,3.6)$ & 1.0 \\
\hline IL-8 (CXCL8) & $5.7(1.7,19)$ & $2.4(1.1,5.5)$ & $2.3(0.9,5.6)$ & 1.0 & $1.2(0.2,8.7)$ & $2.1(0.9,4.8)$ & $1.7(0.7,4.5)$ & 1.0 \\
\hline MCP-1 (CCL2) & $2.8(0.8,10)$ & $1.3(0.6,3.1)$ & $2.6(1.1,6.3)$ & 1.0 & $1.3(0.2,6.6)$ & $1.0(0.4,2.5)$ & $1.5(0.6,3.9)$ & 1.0 \\
\hline MCP-4 (CCL13) & $1.9(0.4,8.2)$ & $0.8(0.3,2.0)$ & $2.6(1.1,6.1)$ & 1.0 & $1.1(0.2,5.8)$ & $0.6(0.2,1.5)$ & $1.4(0.5,3.6)$ & 1.0 \\
\hline MIP-1 $\beta$ (CCL4) & $6.3(1.4,29)$ & $2.0(0.95,4.3)$ & $2.6(1.1,6.1)$ & 1.0 & $5.3(1.2,24)$ & $1.5(0.7,3.2)$ & $1.1(0.4,3.1)$ & 1.0 \\
\hline RANTES (CCL5) & $5.6(0.9,36)$ & $1.3(0.6,2.8)$ & $2.5(1.1,5.5)$ & 1.0 & $1.2(0.1,20)$ & $0.7(0.3,1.7)$ & $1.3(0.5,3.3)$ & 1.0 \\
\hline I-TAC (CXCL11) & $2.3(0.4,13)$ & $1.0(0.4,2.4)$ & $2.5(1.1,5.7)$ & 1.0 & $2.8(0.7,11)$ & $0.4(0.1,1.3)$ & $1.0(0.3,2.7)$ & 1.0 \\
\hline ICAM-1 (CD54) & $4.1(1.2,14)$ & $2.6(1.2,5.6)$ & $2.8(1.1,6.8)$ & 1.0 & $1.4(0.3,7.1)$ & $1.5(0.6,3.4)$ & $1.6(0.6,4.2)$ & 1.0 \\
\hline ICAM-3 (CD50) & $1.7(0.2,13)$ & $1.6(0.8,3.3)$ & $3.1(1.4,6.9)$ & 1.0 & $1.2(0.2,8.9)$ & $0.6(0.2,1.6)$ & $1.3(0.5,3.4)$ & 1.0 \\
\hline VCAM-1 (CD106) & $3.3(0.7,16)$ & $1.4(0.7,3.1)$ & $2.7(1.2,6.2)$ & 1.0 & $2.0(0.4,11)$ & $0.8(0.3,1.8)$ & $1.2(0.5,3.2)$ & 1.0 \\
\hline E-SEL (CD62E) & $4.4(1.2,17)$ & $2.2(1.03,4.7)$ & $2.7(1.1,6.5)$ & 1.0 & $3.1(0.8,12)$ & $1.5(0.7,3.4)$ & $1.2(0.4,3.4)$ & 1.0 \\
\hline MMP-1 & $2.7(0.5,16)$ & $0.7(0.3,1.8)$ & $2.3(1.04,5.3)$ & 1.0 & $2.3(0.4,14)$ & $0.6(0.3,1.5)$ & $1.2(0.4,3.0)$ & 1.0 \\
\hline MMP-9 & $1.3(0.1,21)$ & $0.8(0.3,2.1)$ & $2.6(1.2,5.6)$ & 1.0 & $1.2(0.1,19)$ & $0.7(0.3,1.8)$ & $1.4(0.6,3.3)$ & 1.0 \\
\hline VEGF & $1.3(0.1,21)$ & $1.5(0.7,3.0)$ & $3.0(1.3,6.7)$ & 1.0 & $0.8(0.1,12)$ & $0.4(0.2,1.1)$ & $1.3(0.5,3.2)$ & 1.0 \\
\hline VEGF-R1 & $2.1(0.5,8.2)$ & $0.6(0.2,1.8)$ & $2.5(1.1,5.9)$ & 1.0 & $2.7(0.8,8.9)$ & $0.8(0.3,2.0)$ & $0.9(0.3,2.8)$ & 1.0 \\
\hline VEGF-R2 & $2.2(0.4,12)$ & $2.4(1.2,5.0)$ & $3.4(1.5,7.8)$ & 1.0 & $2.2(0.5,9.9)$ & $1.0(0.4,2.4)$ & $1.2(0.5,3.4)$ & 1.0 \\
\hline IGFBP-1 & $4.9(1.7,15)$ & $2.0(0.9,4.8)$ & $2.0(0.8,5.3)$ & 1.0 & $1.9(0.5,7.3)$ & $0.9(0.3,2.6)$ & $1.2(0.4,3.5)$ & 1.0 \\
\hline
\end{tabular}

The logistic regression models are adjusted for gestational age (23-24, 25-26,27 wk) and sex. The sample consisted of children who had a Gross Motor Function Classification System score $<1$ and had proteins measured on two separate days (maximum $n=805$ ). The referent group consists of children who were not growth restricted at birth and who did not have 2 d of elevated concentrations of the protein listed on the left. Bold indicates odds ratios significantly $>1.0(P<0.01)$.

CRP, C-reactive protein; E-SEL, E-selectin; ICAM, intercellular adhesion molecule; IGFBP, insulin growth factor binding protein-1; IL-6R, interleukin-6 receptor; I-TAC, interferon-inducible T-cell a-chemoattractant; MCP, monocyte chemotactic protein; MIP-1 $\beta$, macrophage inflammatory protein-1 $\beta$; MMP, matrix metalloproteinase; MPO, myeloperoxidase; RANTES, regulated upon activation, normal T-cell expressed, and (presumably) secreted protein; SAA, serum amyloid A; TNF, tumor necrosis factor; VCAM-1, vascular cell adhesion molecule-1; VEGF, vascular endothelial growth factor.

aBayley Scales of Infant Development Mental Development Index when Gross Motor Function Classification System score <1. 'birth weight below the 10th percentile for gestational age.

(endotoxin) among growth-restricted rats than among rats that were not growth restricted (14). In this model, one hit (the inflammatory stimulus, lipopolysaccharide) is followed by damage, but a previous hit (a process associated with SGA or perhaps multiple processes) allows the second hit to result in greater damage than if the first hit had not occurred. No abnormalities were seen in the brains of the growth-restricted rat pups not exposed to the inflammatory stimulus. Thus, this model qualifies for the concept of sensitization, which like preconditioning requires that the first exposure alone not produce damage (27). This is in contrast to some two-hit models that have the first hit produce some damage and the second hit enhance or amplify the damage (28-32).
Why Do Two-Hit Models Apply to an MDI $<55$ But Not to an MDI Between 55 and 69?

Our two-hit models apply to an MDI $>3$ SDs below the mean but not to the less severe reduction of an MDI between 2 and 3 SDs below the mean. We are not sure why what we see prominently for an extreme outcome is not seen less extremely for a less extreme outcome. One possibility is that the risk factors for MDI $<55$ differ from those for MDI 55-69. Another possibility is that what we see are the consequences of a threshold effect.

\section{SGA, Inflammation, and Adult Diseases}

The presumed late consequences of SGA, including adult-onset diabetes, obesity, hypertension, and atherosclerotic disease, are 
associated with inflammation (33). We did not see evidence of inflammation on the first postnatal day (21). Perhaps what we saw just a few weeks later is the earliest indication of an intense inflammatory proclivity that accounts for some of the adult diseases associated with SGA.

\section{Epigenetic Mechanisms}

Epigenetic mechanisms, which can influence phenotypic plasticity without altering genomic DNA, have been invoked to explain a wide variety of SGA consequences, including adult diseases (33), as well as inflammatory processes associated with neonatal brain damage (34). If the processes associated with growth restriction in utero account for some of the brain's sensitivity to postnatal inflammation, then therapies that interfere with epigenetic processes might protect the brain (34).

\section{Choice of Potential Confounders}

Our search for potential confounders of the relationships among SGA, systemic inflammation, and low MDI was especially difficult because some of them might be in the causal chain leading from being SGA to low MDI. For example, the majority of SGA infants were born to women who had severe preeclampsia or were delivered for fetal indications. Moreover, magnesium was given for seizure prophylaxis almost exclusively to women with severe preeclampsia. Indication for delivery and duration of ventilation neither added independent information about the risk of an MDI $<55$ nor altered the odds ratios associated with SGA or any indicator of systemic inflammation. On the other hand, bacteremia contributed supplemental information about the risk of a very low MDI without altering the contributions of SGA and indicators of systemic inflammation. These observations support our decision to present analyses without adjusting for these variables.

We did adjust for gestational age because this is probably our best indicator of unidentified correlates of maturity (35). We also adjusted for sex because being SGA usually predicts low MDI differently in each sex (36).

\section{Implications}

If our findings are replicated, they will be an example of a two-hit model of brain damage in humans. Two implications follow from our observations. First, our findings might be simply explained as an example of an additive model of increased risk, although consideration should be given to the possibility that the consequences of being SGA and having systemic inflammation are more than additive. Second, effective intervention to improve outcome among SGA infants might be possible even after delivery.

\section{Conclusions}

Very preterm SGA newborns appear to be at an increased risk of severely limited mental development, especially if they experienced early postnatal systemic inflammation.

\section{METHODS}

The Extremely Low Gestational Age Newborns (ELGAN) study was designed to identify characteristics and exposures that increase the risk of structural and functional neurologic disorders in ELGAN. During the years 2002-2004, women delivering before $28 \mathrm{wk}$ gestation at one of 14 participating institutions were asked to enroll in the study. Each institution's review board approved the enrollment and consent procedures and documents. A full description of the methods is provided elsewhere (37). Here, we focus on those most relevant to these analyses.

The sample for this report consists of 805 newborns for whom we had information about protein concentrations on at least two of the three protocol days (days 1, 7, and 14), who had a developmental assessment at the age of 2 y postterm equivalent, and who did not have a motor handicap that might interfere with the assessments of cognition (Table 1).

After delivery, a trained research nurse interviewed each mother in her native language using a structured data collection form and following procedures defined in a manual. Shortly after the mother's discharge, the research nurse reviewed the maternal chart using a second structured data collection form. The medical record was relied on for events following the admission.

Definitions of Pregnancy Disorders That Led to Preterm Delivery The clinical circumstances that led to each maternal admission and ultimately to each preterm delivery were operationally defined using both data from the maternal interview and data abstracted from the medical record (38). Each mother-infant pair was assigned to the category that described the primary reason for the preterm delivery. Preterm labor was defined as progressive cervical dilation with regular contractions and intact membranes. The diagnosis of preterm, premature rupture of fetal membranes was defined as the presence of vaginal pooling with either documented nitrazine-positive testing or ferning before regular uterine activity. Preeclampsia was defined as new-onset hypertension and proteinuria of sufficient severity to warrant delivery for either a maternal or fetal indication. A diagnosis of cervical insufficiency was made when a woman presented with cervical dilation of $>2 \mathrm{~cm}$ but no membrane rupture or perceived uterine activity. Placental abruption was defined as appreciable vaginal bleeding (either documented in the medical record or a postpartum hematocrit $<24 \%$ ) and a clinical diagnosis of placental abruption in the absence of cervical change. Presentations under the category of fetal indication included severe intrauterine growth restriction on the basis of antepartum ultrasound examination, nonreassuring fetal testing, oligohydramnious, and Doppler abnormalities of umbilical cord blood flow.

\section{Placenta Bacteriology and Morphology}

Delivered placentas were placed in a sterile examination basin and transported to a sampling room, where they were biopsied under sterile conditions and flash-frozen. Eighty-two percent of the samples were obtained within $1 \mathrm{~h}$ of delivery. At a later time, the samples were allowed to thaw at room temperature, a portion $\sim 1 \mathrm{~cm}^{2}$ was removed and weighed, then diluted 1:10 with sterile phosphate-buffered saline, and homogenized. Aliquots were plated on selective and nonselective media, including prereduced Brucella-base agar with 5\% sheep blood enriched with hemin and vitamin K1, tryptic soy agar with $5 \%$ sheep blood, chocolate agar, and A-7 agar. After incubation, the various colony types were enumerated, isolated, and identified by established criteria.

For histologic evaluation, representative sections were taken from all abnormal areas as well as routine sections from the umbilical cord and a membrane roll, and full-thickness sections from the center and a paracentral zone of the placental disc. After training to minimize observer variability, study pathologists examined the slides for histologic characteristics listed on a standardized data form they helped create $(15,16)$. Briefly, infarcts and intervillous fibrin, fetal stem vessel thrombosis, and decidual hemorrhage and fibrin deposition consistent with abruption were coded as present or absent. Chorionic villi were scored for syncytial knots (none, occasional, or increased).

At the chorionic plate of the disc, grade 3 acute inflammation was defined as neutrophils up to amnionic epithelium, and stage 3 was defined as $>20$ neutrophils/20x. Grade 3 inflammation of the external membranes, as well as of the chorion/decidua, required numerous large or confluent foci of the neutrophils.

Inflammation in the umbilical cord was graded from 0 to 5 . Grade 3 required neutrophils in the perivascular Wharton's jelly; grade 4 
required panvasculitis and umbilical cord vasculitis extending deep into the Wharton's jelly; and grade 5 required a "halo lesion" (ring of precipitate in the Wharton's jelly encircling each vessel). Neutrophilic infiltration into fetal stem vessels in the chorionic plate required that neutrophils appeared to have migrated toward the amnionic cavity.

\section{Newborn Variables}

The gestational age estimates were based on a hierarchy of the quality of available information. Most desirable were estimates based on the dates of embryo retrieval or intrauterine insemination or fetal ultrasound before the 14th wk (62\%). When these were not available, reliance was placed sequentially on a fetal ultrasound at 14 or more weeks $(29 \%)$, last menstrual period without fetal ultrasound (7\%), and gestational age recorded in the log of the neonatal intensive care unit (1\%).

A newborn was considered to be SGA if the birth weight was in the lowest decile of the birth weight distribution in an external standard (19). This is equivalent to a birth weight $>1.28$ SDs below the mean in a referent population.

Documented early bacteremia was defined as recovery of an organism from blood drawn during the first postnatal week, and late bacteremia as recovery of an organism from blood drawn during the second, third, or fourth postnatal week. Presumed bacteremia was defined as culture negative but the clinician ordered antibiotics for $>72 \mathrm{~h}$.

The lowest and highest arterial blood measurements of $\mathrm{PaO}_{2}, \mathrm{PCO}_{2}$, and $\mathrm{pH}$ on postnatal days 1,2 , and 3 were routinely recorded. We identified ELGAN whose blood gas measurements were in the extreme quartile for gestational age on at least 2 of the $3 \mathrm{~d}$.

Information about the mode of ventilation was collected for each day during the first $7 \mathrm{~d}$ and weekly thereafter until $28 \mathrm{~d}$. ELGAN were classified into three mutually exclusive groups: those with a consistently low fraction of inspired oxygen $\left(\mathrm{FiO}_{2}\right)\left(\right.$ an $\mathrm{FiO}_{2}<0.23$ each day between postnatal days 3 and 7 and receiving $\mathrm{FiO}_{2} \leq 0.25$ on day 14), those with pulmonary deterioration ( $\mathrm{an} \mathrm{FiO}_{2}<0.23$ on any day between days 3 and 7 and receiving $\mathrm{FiO}_{2}>0.25$ on day 14 ), and those with early and persistent pulmonary dysfunction ( $\mathrm{an} \mathrm{FiO}_{2} \geq 0.23$ on all days between 3 and 7 $\mathrm{d}$ of life and receiving $\mathrm{FiO}_{2}>0.25$ on day 14) (5).

\section{Developmental Assessment at 24 Mo}

Overall, $91 \%$ of surviving children returned for a developmental assessment close to the time when she/he would be 24 mo of corrected age. Of these children, $77 \%$ had their examination within the range of 23.5-27.9 mo. Most others were examined before 23.5 mo.

Certified examiners administered and scored the Bayley Scales of Infant Development-2nd edn (39). We chose as our main outcome a MDI $<55$ because it is three SDs below the expected mean and therefore constitutes a severe impairment, and because the predictive ability of an MDI $<55$ is higher than that of a score $<70$, which is two SDs below the expected mean (40). Because some MDI test items require intact motor function, we excluded all infants with significantly impaired gross motor function, defined as an inability to walk independently (a Gross Motor Function Classification System score $\geq 1$ ), regardless of whether or not they were given a cerebral palsy diagnosis.

\section{Blood Spot Collection}

After blood was collected for clinical indications, drops were blotted on filter paper on the 1st postnatal day (range: $1-3 \mathrm{~d}$ ), the 7 th postnatal day (range: 5-8 d), and the 14th postnatal day (range: $12-15 \mathrm{~d}$ ). Dried blood spots were stored at $-70^{\circ} \mathrm{C}$ in sealed bags with desiccant until processed. All references to protein concentrations refer to the three samples obtained on or about days 1, 7, and 14 .

\section{Protein Measurement}

Details about elution of proteins from blood spots and measurement of the proteins with the Meso Scale Discovery electrochemiluminescence system are provided elsewhere (22). Interassay variations are invariably $<20 \%$. Measurements of each protein were normalized to milligrams of total protein.

The Laboratory of Genital Tract Biology of the Department of Obstetrics, Gynecology and Reproductive Biology at Brigham and Women's Hospital, Boston, measured the following 25 proteins: IL- $1 \beta$, IL-6, IL-6 receptor, TNF- $\alpha$, TNF- $\alpha$ receptor-1, TNF- $\alpha$ receptor-2),
IL-8 (CXCL8), monocyte chemotactic protein-1 (CCL2), monocyte chemotactic protein-4(CCL13), macrophage inflammatory protein-1 $\beta$ (CCL4), regulated upon activation, normal T-cell expressed, and (presumably) secreted protein (CCL5), interferon-inducible T-cell a-chemoattractant (CXCL11), ICAM-1 (CD54), ICAM-3 (CD50), vascular cell adhesion molecule-1 (CD106), E-selectin (CD62E), matrix metalloproteinase-1, matrix metalloproteinase-9, C-reactive protein), SAA, myeloperoxidase, vascular endothelial growth factor, vascular endothelial growth factor receptor-1, vascular endothelial growth factor receptor-2, and insulin growth factor binding protein-1.

In previous analyses in this sample, protein elevations in the top quartile (for gestational age and postnatal day) on two separate days provided considerably more discriminating risk information than did elevations on just one day (10-12). Thus, our indicator of postnatal systemic inflammation is a concentration of an inflammation-related protein in the top quartile that persisted or recurred.

\section{Data Analysis}

We evaluated the following hypotheses about the risk of a low MDI, defined as either a very low MDI (i.e., $<55$ ) or a moderately low MDI (i.e., between 55 and 69). First, as compared with ELGAN who were neither SGA nor had recurrent/persistent postnatal systemic inflammation, those who had both the characteristics were more likely to have a low MDI 2 y later. Second, among ELGAN who were SGA, those who had recurrent/persistent early postnatal systemic inflammation were much more likely to have a low MDI 2 y later. Third, among ELGAN who had recurrent/persistent early postnatal systemic inflammation, those who were SGA were more likely to have a low MDI 2 y later.

Because our outcomes of interest (an MDI $<55$ and an MDI between 55 and 69) are mutually exclusive and each is appropriately compared with the same referent group (MDI $\geq 70$ ), we created multinomial logistic regression models. This allowed us to calculate the odds ratios and $99 \%$ confidence intervals in separate models for each protein that included variables for SGA, persistent/recurrent protein concentrations in the top quartile on two days a week apart, as well as variables for gestational age (23-24, 25-26, and $27 \mathrm{wk})$ and sex.

\section{ACKNOWLEDGMENTS}

The authors gratefully acknowledge the contributions of their subjects and their subjects' families, as well as the following ELGAN Study colleagues for all their contributions: Bhavesh L. Shah, Baystate Medical Center, Springfield, MA; Camilia Martin, Beth Israel Deaconess Medical Center, Boston, MA; Linda Van Marter, Brigham \& Women's Hospital, Boston, MA; Robert Insoft, Massachusetts General Hospital, Boston, MA; Francis Bednarek, U Mass Memorial Health Center, Worcester, MA; Olaf Dammann, John Fiascone, Cynthia Cole, Tufts Medical Center, Boston, MA; Richard A. Ehrenkranz, Yale-New Haven Children's Hospital, New Haven, CT; Stephen C. Engelke, University Health Systems of Eastern Carolina, Greenville, NC; Carl Bose, University of North Carolina, Chapel Hill, NC; Mariel Poortenga, DeVos Children's Hospital, Grand Rapids, Ml; Padima Karna, Sparrow Hospital, Lansing, Ml; Michael D. Schreiber, University of Chicago Hospital, Chicago, IL; Daniel Batton, William Beaumont Hospital, Royal Oak, Ml; Greg Pavlov, Frontier Science and Technology Research Foundation, Amherst, NY; Deborah Hirtz, National Institute of Neurological Disorders and Stroke, Bethesda, MD.

\section{STATEMENT OF FINANCIAL SUPPORT}

This study was supported by a cooperative agreement with the National Institute of Neurological Disorders and Stroke (grants 5U01NS040069-05 and 2R01NS040069-06A2) and a center grant award from the National Institute of Child Health and Human Development (grant 5P30HD018655-28).

Disclosure: The authors declared no conflict of interest.

\section{REFERENCES}

1. Hutcheon JA, Platt RW. The missing data problem in birth weight percentiles and thresholds for "small-for-gestational-age". Am J Epidemiol 2008; 167:786-92.

2. Padilla N, Falcón C, Sanz-Cortés M, et al. Differential effects of intrauterine growth restriction on brain structure and development in preterm infants: a magnetic resonance imaging study. Brain Res 2011;1382:98-108. 
3. Guellec I, Lapillonne A, Renolleau S, et al.; EPIPAGE Study Group. Neurologic outcomes at school age in very preterm infants born with severe or mild growth restriction. Pediatrics 2011;127:e883-91.

4. Morsing E, Asard M, Ley D, Stjernqvist K, Marsál K. Cognitive function after intrauterine growth restriction and very preterm birth. Pediatrics 2011;127:e874-82.

5. Graham EM, Holcroft CJ, Rai KK, Donohue PK, Allen MC. Neonatal cerebral white matter injury in preterm infants is associated with culture positive infections and only rarely with metabolic acidosis. Am J Obstet Gynecol 2004;191:1305-10.

6. Glass HC, Bonifacio SL, Chau V, et al. Recurrent postnatal infections are associated with progressive white matter injury in premature infants. Pediatrics 2008;122:299-305.

7. Shah DK, Doyle LW, Anderson PJ, et al. Adverse neurodevelopment in preterm infants with postnatal sepsis or necrotizing enterocolitis is mediated by white matter abnormalities on magnetic resonance imaging at term. J Pediatr 2008;153:170-5, 175.e1.

8. Martin CR, Dammann O, Allred EN, et al. Neurodevelopment of extremely preterm infants who had necrotizing enterocolitis with or without late bacteremia. J Pediatr 2010;157:751-6.e1.

9. van der Ree M, Tanis JC, Van Braeckel KN, Bos AF, Roze E. Functional impairments at school age of preterm born children with late-onset sepsis. Early Hum Dev 2011;87:821-6.

10. Leviton A, Kuban KC, Allred EN, Fichorova RN, O'Shea TM, Paneth N; ELGAN Study Investigators. Early postnatal blood concentrations of inflammation-related proteins and microcephaly two years later in infants born before the 28th post-menstrual week. Early Hum Dev 2011;87: 325-30.

11. Leviton A, Kuban K, O'Shea TM, et al. The relationship between early concentrations of 25 blood proteins and cerebral white matter injury in preterm newborns: the ELGAN study. J Pediatr 2011;158:897-903.e1-5.

12. O'Shea TM, Allred EN, Kuban KC, et al.; Extremely Low Gestational Age Newborn (ELGAN) Study Investigators. Elevated concentrations of inflammation-related proteins in postnatal blood predict severe developmental delay at 2 years of age in extremely preterm infants. J Pediatr 2012;160:395-401.e4.

13. Resch B, Neubauer K, Hofer N, et al. Episodes of hypocarbia and earlyonset sepsis are risk factors for cystic periventricular leukomalacia in the preterm infant. Early Hum Dev 2012;88:27-31.

14. Campbell LR, Pang Y, Ojeda NB, Zheng B, Rhodes PG, Alexander BT. Intracerebral lipopolysaccharide induces neuroinflammatory change and augmented brain injury in growth-restricted neonatal rats. Pediatr Res 2012;71:645-52.

15. Arnold CC, Kramer MS, Hobbs CA, McLean FH, Usher RH. Very low birth weight: a problematic cohort for epidemiologic studies of very small or immature neonates. Am J Epidemiol 1991;134:604-13.

16. Sackett DL. Bias in analytic research. J Chronic Dis 1979;32:51-63.

17. Kuban KCK, Allred EN, O'Shea TM, et al. An algorithm for diagnosing and classifying cerebral palsy in young children. J Pediat 2008;153:466-72. e461.

18. Fichorova RN, Richardson-Harman N, Alfano M, et al. Biological and technical variables affecting immunoassay recovery of cytokines from human serum and simulated vaginal fluid: a multicenter study. Anal Chem 2008;80:4741-51.

19. Fichorova RN, Onderdonk AB, Yamamoto H, et al.; Extremely Low Gestation Age Newborns (ELGAN) Study Investigators. Maternal microbe-specific modulation of inflammatory response in extremely low-gestationalage newborns. MBio 2011;2:e0280-10.

20. Hecht JL, Fichorova RN, Tang VF, Allred EN, McElrath TF, Leviton A; Elgan Study Investigators. Relationship Between Neonatal Blood Protein
Concentrations and Placenta Histologic Characteristics in Extremely Low GA Newborns. Pediatr Res 2011;69:68-73.

21. McElrath TF, Fichorova RN, Allred EN, et al.; ELGAN Study Investigators. Blood protein profiles of infants born before 28 weeks differ by pregnancy complication. Am J Obstet Gynecol 2011;204:418.e1-12.

22. Leviton A, Fichorova R, Yamamoto Y, et al. Inflammation-related proteins in the blood of extremely low gestational age newborns. The contribution of inflammation to the appearance of developmental regulation. Cytokine 2011;53:66-73.

23. Ramma W, Ahmed A. Is inflammation the cause of pre-eclampsia? Biochem Soc Trans 2011;39:1619-27.

24. Cemgil Arikan D, Aral M, Coskun A, Ozer A. Plasma IL-4, IL-8, IL-12, interferon- $\gamma$ and CRP levels in pregnant women with preeclampsia, and their relation with severity of disease and fetal birth weight. J Matern Fetal Neonatal Med 2012;25:1569-73.

25. Sugimoto J, Romani AM, Valentin-Torres AM, et al. Magnesium decreases inflammatory cytokine production: a novel innate immunomodulatory mechanism. J Immunol 2012;188:6338-46.

26. Pallotto EK, Kilbride HW. Perinatal outcome and later implications of intrauterine growth restriction. Clin Obstet Gynecol 2006;49:257-69.

27. Eklind S, Mallard C, Leverin AL, et al. Bacterial endotoxin sensitizes the immature brain to hypoxic-ischaemic injury. Eur J Neurosci 2001;13:1101-6.

28. Dammann O, Brinkhaus MJ, Bartels DB, et al. Immaturity, perinatal inflammation, and retinopathy of prematurity: a multi-hit hypothesis. Early Hum Dev 2009;85:325-9.

29. Adén U, Favrais G, Plaisant F, et al. Systemic inflammation sensitizes the neonatal brain to excitotoxicity through a pro-/anti-inflammatory imbalance: key role of TNFalpha pathway and protection by etanercept. Brain Behav Immun 2010;24:747-58.

30. Williamson LL, Sholar PW, Mistry RS, Smith SH, Bilbo SD. Microglia and memory: modulation by early-life infection. J Neurosci 2011;31:15511-21.

31. Orman MA, Ierapetritou MG, Berthiaume F, Androulakis IP. The dynamics of the early inflammatory response in double-hit burn and sepsis animal models. Cytokine 2011;56:494-502.

32. Patten IS, Rana S, Shahul S, et al. Cardiac angiogenic imbalance leads to peripartum cardiomyopathy. Nature 2012;485:333-8.

33. Joss-Moore LA, Lane RH. The developmental origins of adult disease. Curr Opin Pediatr 2009;21:230-4.

34. Hagberg H, Gressens P, Mallard C. Inflammation during fetal and neonatal life: implications for neurologic and neuropsychiatric disease in children and adults. Ann Neurol 2012;71:444-57.

35. Leviton A, Blair E, Dammann O, Allred E. The wealth of information conveyed by gestational age. J Pediatr 2005;146:123-7.

36. Streimish IG, Ehrenkranz RA, Allred EN, et al.; ELGAN Study Investigators. Birth weight- and fetal weight-growth restriction: impact on neurodevelopment. Early Hum Dev 2012;88:765-71.

37. O'Shea TM, Allred EN, Dammann O, et al.; ELGAN study Investigators. The ELGAN study of the brain and related disorders in extremely low gestational age newborns. Early Hum Dev 2009;85:719-25.

38. McElrath TF, Hecht JL, Dammann O, et al.; ELGAN Study Investigators. Pregnancy disorders that lead to delivery before the 28th week of gestation: an epidemiologic approach to classification. Am J Epidemiol 2008;168:980-9.

39. Bayley N. Bayley Scales of Infant Development-II. San Antonio, TX: Psychological Corporation, 1993.

40. Roberts G, Anderson PJ, Doyle LW; Victorian Infant Collaborative Study Group. The stability of the diagnosis of developmental disability between ages 2 and 8 in a geographic cohort of very preterm children born in 1997. Arch Dis Child 2010;95:786-90. 